\title{
Timed Unfoldings For Networks of Timed Automata
}

Patricia Bouyer $^{1} \quad$ Serge Haddad ${ }^{2} \quad$ Pierre-Alain Reynier ${ }^{1}$

${ }^{1}$ LSV, CNRS \& ENS Cachan, France

${ }^{2}$ LAMSADE, CNRS \& Univ. Paris Dauphine, France

ULB, Bruxelles, 21 décembre 2006 presented at ATVA'06 


\section{Motivations}

The general framework is this of model-checking :

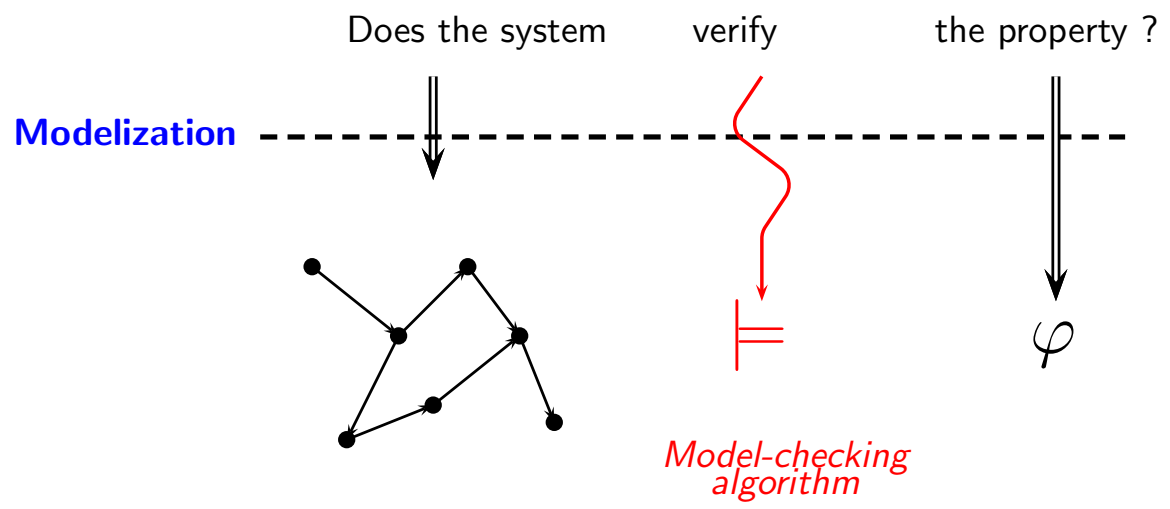




\section{Motivations}

\section{Our objective:}

$\rightarrow$ Apply partial order techniques to timed systems.

In this work:

- Partial order techniques $=$ unfoldings

- Timed systems $=$ Networks of timed automata (NTA)

Classical difficulties:

- merge time and concurrency

- handle urgency (invariants in TA) 


\section{Related Work}

Partial order methods for NTA:

- Local semantics of time [Yi et al 98, Minea 99]

- Mazurkiewicz traces [Niebert et al 04, 06]

Unfoldings for time Petri nets:

- Unfolding of the underlying Petri net [Lilius 98]

- Proved to be "locally infeasible" [Aura \& Lilius 00]

- Unfolding with discrete time [Fleishback \& Steino 02]

- General case, introducing read arcs [Chatain \& Jard 06]. 
(1) Generalities

- Networks of timed automata

- Unfolding of discrete-event systems

(2) Discrete Structure of our Unfolding

- Clocks as shared variables

- Invariants

- An example

(3) Adding Time

- Timed non-branching process

- A first timed unfolding

- Computation via local zones

(4) Finite and complete prefix

(5) Conclusion 
(1) Generalities

- Networks of timed automata

- Unfolding of discrete-event systems

Discrete Structure of our Unfolding

- Clocks as shared variables

- Invariants

- An example

Adding Time

- Timed non-branching process

- A first timed unfolding

- Computation via local zones

Finite and complete prefix 


\section{Network of Timed Automata (NTA)}

A NTA is a finite set of processes, defined as TA, together with a synchronization function. We allow:

- invariants,

- shared clocks,

- $n$-ary synchronizations.

Configuration $=$ a vector of discrete locations + a clock valuation

Example:

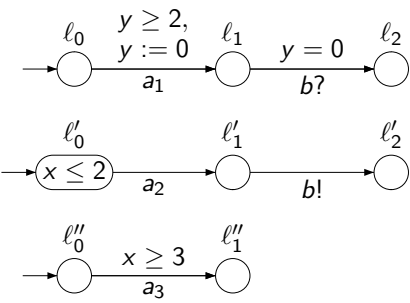




\section{Network of Timed Automata (NTA)}

$$
\text { x) }
$$

Example:

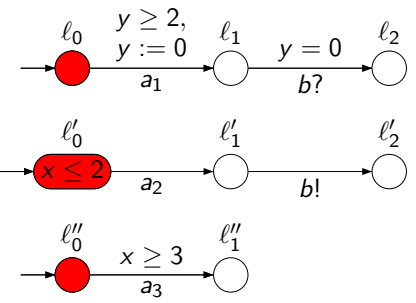$$
f:\left\{\begin{array}{rlr}
\left(a_{1}, \perp, \perp\right) & \mapsto a_{1} \\
\left(\perp, a_{2}, \perp\right) & \mapsto a_{2} \\
\left(\perp, \perp, a_{3}\right) & \mapsto a_{3} \\
(b ?, b !, \perp) & \mapsto b
\end{array}\right.
$$ 


\section{Network of Timed Automata (NTA)}

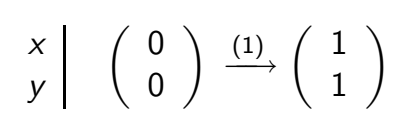

Example:

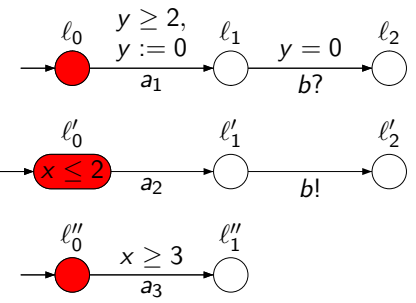$$
f:\left\{\begin{array}{rlr}
\left(a_{1}, \perp, \perp\right) & \mapsto a_{1} \\
\left(\perp, a_{2}, \perp\right) & \mapsto a_{2} \\
\left(\perp, \perp, a_{3}\right) & \mapsto a_{3} \\
(b ?, b !, \perp) & \mapsto b
\end{array}\right.
$$ 


\section{Network of Timed Automata (NTA)}

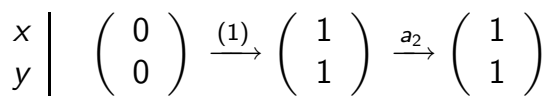

Example:

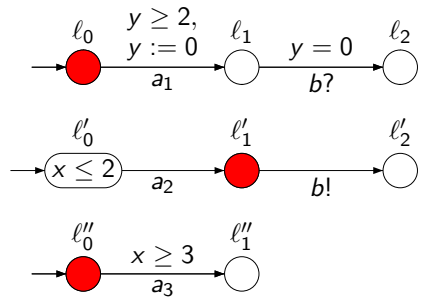$$
f:\left\{\begin{array}{rlr}
\left(a_{1}, \perp, \perp\right) & \mapsto a_{1} \\
\left(\perp, a_{2}, \perp\right) & \mapsto a_{2} \\
\left(\perp, \perp, a_{3}\right) & \mapsto a_{3} \\
(b ?, b !, \perp) & \mapsto b
\end{array}\right.
$$ 


\section{Network of Timed Automata (NTA)}

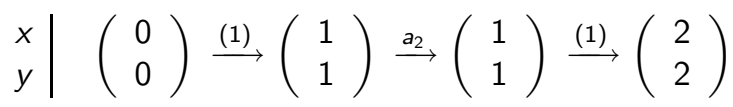

Example:

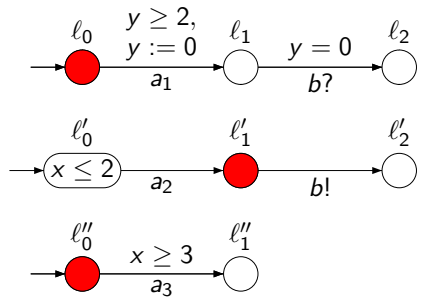$$
f:\left\{\begin{aligned}
\left(a_{1}, \perp, \perp\right) & \mapsto a_{1} \\
\left(\perp, a_{2}, \perp\right) & \mapsto a_{2} \\
\left(\perp, \perp, a_{3}\right) & \mapsto a_{3} \\
(b ?, b !, \perp) & \mapsto b
\end{aligned}\right.
$$ 


\section{Network of Timed Automata (NTA)}

$$
\begin{gathered}
\left.\begin{array}{c}
x \\
y
\end{array}\right)\left(\begin{array}{l}
0 \\
0
\end{array}\right) \stackrel{(1)}{\longrightarrow}\left(\begin{array}{l}
1 \\
1
\end{array}\right) \stackrel{a_{2}}{\longrightarrow}\left(\begin{array}{l}
1 \\
1
\end{array}\right) \stackrel{(1)}{\longrightarrow}\left(\begin{array}{l}
2 \\
2
\end{array}\right) \\
\stackrel{a_{1}}{\longrightarrow}\left(\begin{array}{l}
2 \\
0
\end{array}\right)
\end{gathered}
$$

Example:

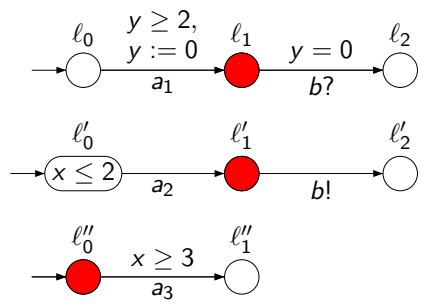

$f:\left\{\begin{aligned}\left(a_{1}, \perp, \perp\right) & \mapsto a_{1} \\ \left(\perp, a_{2}, \perp\right) & \mapsto a_{2} \\ \left(\perp, \perp, a_{3}\right) & \mapsto a_{3} \\ (b ?, b !, \perp) & \mapsto b\end{aligned}\right.$ 


\section{Network of Timed Automata (NTA)}

$$
\begin{gathered}
\left.\begin{array}{c}
x \\
y
\end{array}\right)\left(\begin{array}{l}
0 \\
0
\end{array}\right) \stackrel{(1)}{\longrightarrow}\left(\begin{array}{l}
1 \\
1
\end{array}\right) \stackrel{a_{2}}{\longrightarrow}\left(\begin{array}{l}
1 \\
1
\end{array}\right) \stackrel{(1)}{\longrightarrow}\left(\begin{array}{l}
2 \\
2
\end{array}\right) \\
\stackrel{a_{1}}{\longrightarrow}\left(\begin{array}{l}
2 \\
0
\end{array}\right) \stackrel{b}{\rightarrow}\left(\begin{array}{l}
2 \\
0
\end{array}\right)
\end{gathered}
$$

Example:

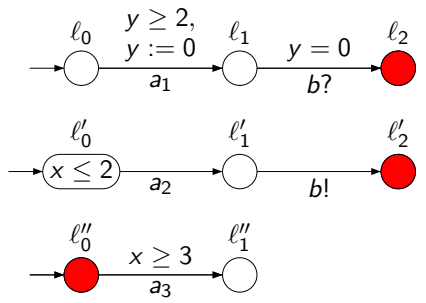$$
f:\left\{\begin{aligned}
\left(a_{1}, \perp, \perp\right) & \mapsto a_{1} \\
\left(\perp, a_{2}, \perp\right) & \mapsto a_{2} \\
\left(\perp, \perp, a_{3}\right) & \mapsto a_{3} \\
(b ?, b !, \perp) & \mapsto b
\end{aligned}\right.
$$ 


\section{Network of Timed Automata (NTA)}

$$
\begin{aligned}
& \begin{array}{l|l}
x \\
y
\end{array} \quad\left(\begin{array}{l}
0 \\
0
\end{array}\right) \stackrel{(1)}{\longrightarrow}\left(\begin{array}{l}
1 \\
1
\end{array}\right) \stackrel{a_{2}}{\longrightarrow}\left(\begin{array}{l}
1 \\
1
\end{array}\right) \stackrel{(1)}{\longrightarrow}\left(\begin{array}{l}
2 \\
2
\end{array}\right) \\
& \stackrel{a_{1}}{\longrightarrow}\left(\begin{array}{l}
2 \\
0
\end{array}\right) \stackrel{b}{\rightarrow}\left(\begin{array}{l}
2 \\
0
\end{array}\right) \stackrel{(1)}{\longrightarrow}\left(\begin{array}{l}
3 \\
1
\end{array}\right)
\end{aligned}
$$

Example:

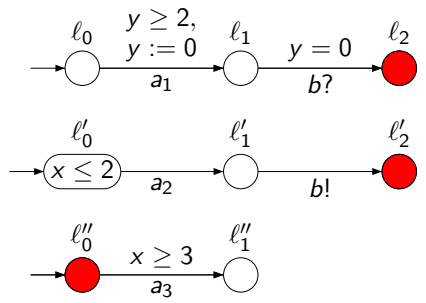

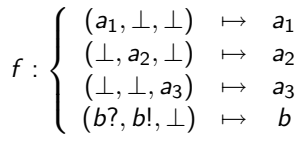




\section{Network of Timed Automata (NTA)}

$$
\begin{aligned}
x & \left(\begin{array}{l}
0 \\
0
\end{array}\right) \stackrel{(1)}{\longrightarrow}\left(\begin{array}{l}
1 \\
1
\end{array}\right) \stackrel{a_{2}}{\longrightarrow}\left(\begin{array}{l}
1 \\
1
\end{array}\right) \stackrel{(1)}{\longrightarrow}\left(\begin{array}{l}
2 \\
2
\end{array}\right) \\
& \stackrel{a_{1}}{\longrightarrow}\left(\begin{array}{l}
2 \\
0
\end{array}\right) \stackrel{b}{\rightarrow}\left(\begin{array}{l}
2 \\
0
\end{array}\right) \stackrel{(1)}{\longrightarrow}\left(\begin{array}{l}
3 \\
1
\end{array}\right) \stackrel{a_{3}}{\longrightarrow}\left(\begin{array}{l}
3 \\
1
\end{array}\right)
\end{aligned}
$$

Example:

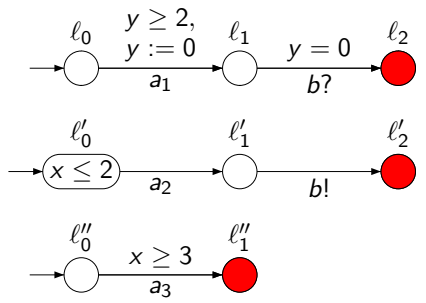$$
f:\left\{\begin{aligned}
\left(a_{1}, \perp, \perp\right) & \mapsto a_{1} \\
\left(\perp, a_{2}, \perp\right) & \mapsto a_{2} \\
\left(\perp, \perp, a_{3}\right) & \mapsto a_{3} \\
(b ?, b !, \perp) & \mapsto b
\end{aligned}\right.
$$ 


\section{Unfolding of discrete-event systems}

Unfolding of a system $=$ net describing its concurrent behaviors.

Usually infinite, but: existence of a finite and complete prefix.

$\rightarrow$ Well kwown for discrete-event systems such as Petri Nets, or networks of automata (McMillan, Esparza et al, ...)

$\rightarrow$ we can decide reachability, transition enabling, deadlocks...

Remark1: Very efficient for safe Petri Nets

Remark2: Networks of Automata give safe Petri Nets!

$\rightarrow$ it remains to handle time! 


\section{Example of the unfolding of a discrete system}
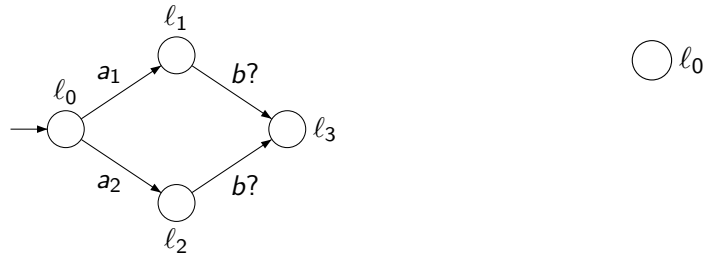

$\bigcirc \ell_{0}^{\prime}$

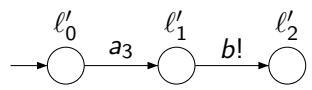

$$
f:\left\{\begin{array}{rlr}
\left(a_{1}, \perp\right) & \mapsto a_{1} \\
\left(\perp, a_{2}\right) & \mapsto & a_{2} \\
\left(\perp, a_{3}\right) & \mapsto & a_{3} \\
(b ?, b !) & \mapsto & b
\end{array}\right.
$$




\section{Example of the unfolding of a discrete system}
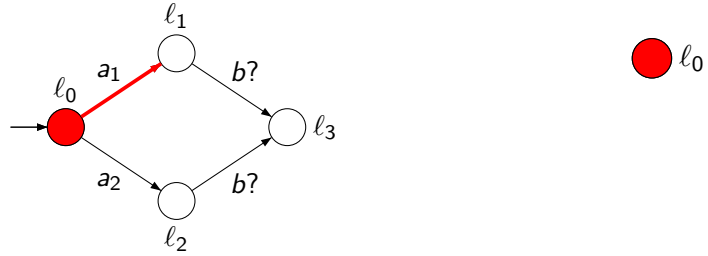

$\bigcirc \ell_{0}^{\prime}$

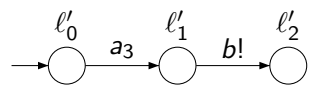

$$
f:\left\{\begin{array}{rlr}
\left(a_{1}, \perp\right) & \mapsto a_{1} \\
\left(\perp, a_{2}\right) & \mapsto a_{2} \\
\left(\perp, a_{3}\right) & \mapsto a_{3} \\
(b ?, b !) & \mapsto b
\end{array}\right.
$$




\section{Example of the unfolding of a discrete system}
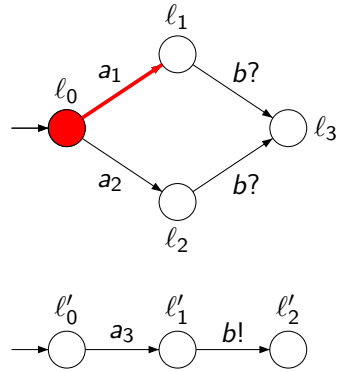

$f:\left\{\begin{aligned}\left(a_{1}, \perp\right) & \mapsto a_{1} \\ \left(\perp, a_{2}\right) & \mapsto a_{2} \\ \left(\perp, a_{3}\right) & \mapsto a_{3} \\ (b ?, b !) & \mapsto b\end{aligned}\right.$

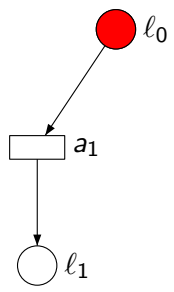

$\bigcirc \ell_{0}^{\prime}$ 


\section{Example of the unfolding of a discrete system}
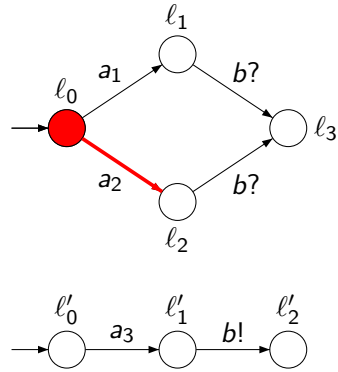

$f:\left\{\begin{aligned}\left(a_{1}, \perp\right) & \mapsto a_{1} \\ \left(\perp, a_{2}\right) & \mapsto a_{2} \\ \left(\perp, a_{3}\right) & \mapsto a_{3} \\ (b ?, b !) & \mapsto b\end{aligned}\right.$

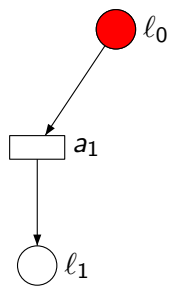

$\bigcirc \ell_{0}^{\prime}$ 


\section{Example of the unfolding of a discrete system}
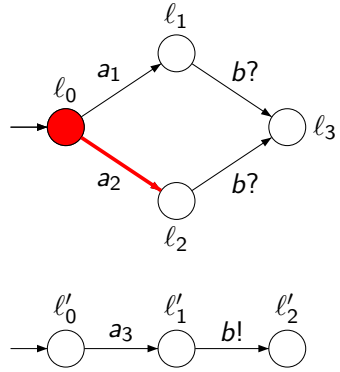

$$
f:\left\{\begin{aligned}
\left(a_{1}, \perp\right) & \mapsto a_{1} \\
\left(\perp, a_{2}\right) & \mapsto a_{2} \\
\left(\perp, a_{3}\right) & \mapsto a_{3} \\
(b ?, b !) & \mapsto b
\end{aligned}\right.
$$

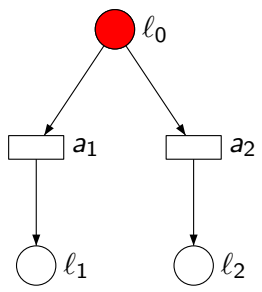

$\bigcirc \ell_{0}^{\prime}$ 


\section{Example of the unfolding of a discrete system}
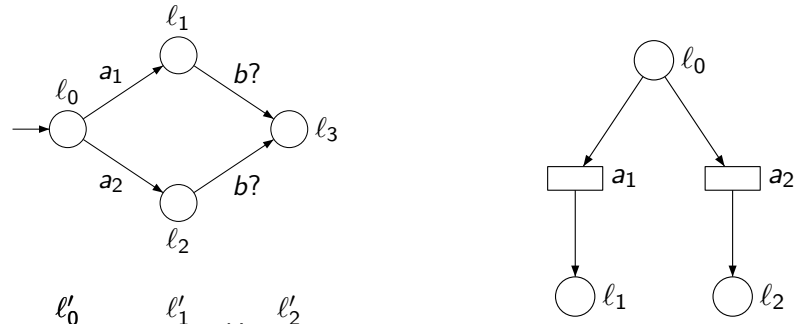

$\ell_{0}^{\prime}$

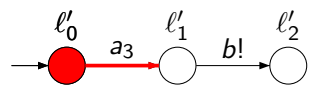

$$
f:\left\{\begin{array}{lll}
\left(a_{1}, \perp\right) & \mapsto a_{1} \\
\left(\perp, a_{2}\right) & \mapsto a_{2} \\
\left(\perp, a_{3}\right) & \mapsto a_{3} \\
(b ?, b !) & \mapsto & b
\end{array}\right.
$$




\section{Example of the unfolding of a discrete system}
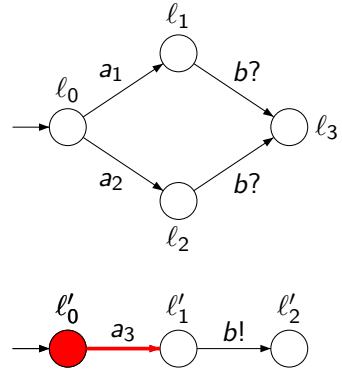

$$
f:\left\{\begin{aligned}
\left(a_{1}, \perp\right) & \mapsto a_{1} \\
\left(\perp, a_{2}\right) & \mapsto a_{2} \\
\left(\perp, a_{3}\right) & \mapsto a_{3} \\
(b ?, b !) & \mapsto b
\end{aligned}\right.
$$
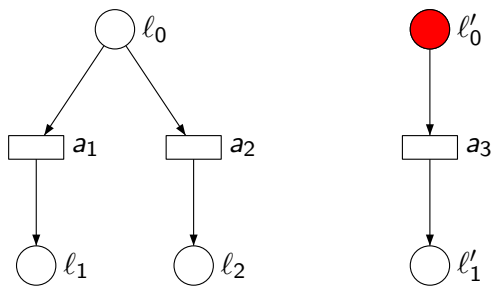


\section{Example of the unfolding of a discrete system}
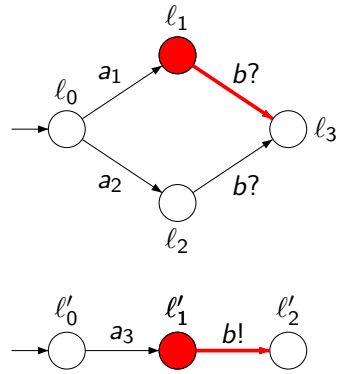

$$
f:\left\{\begin{array}{rlr}
\left(a_{1}, \perp\right) & \mapsto a_{1} \\
\left(\perp, a_{2}\right) & \mapsto a_{2} \\
\left(\perp, a_{3}\right) & \mapsto a_{3} \\
(b ?, b !) & \mapsto b
\end{array}\right.
$$
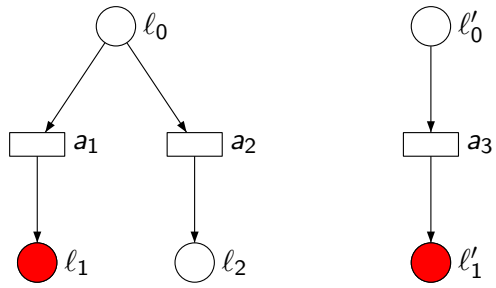


\section{Example of the unfolding of a discrete system}
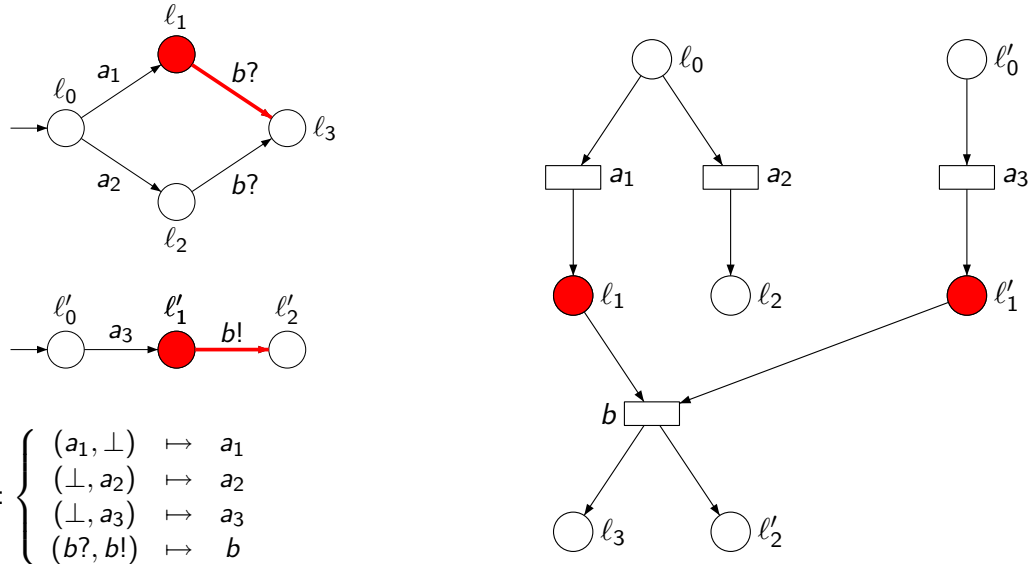

$f:\left\{\begin{array}{rlr}\left(a_{1}, \perp\right) & \mapsto a_{1} \\ \left(\perp, a_{2}\right) & \mapsto a_{2} \\ \left(\perp, a_{3}\right) & \mapsto a_{3} \\ (b ?, b !) & \mapsto & b\end{array}\right.$ 


\section{Example of the unfolding of a discrete system}
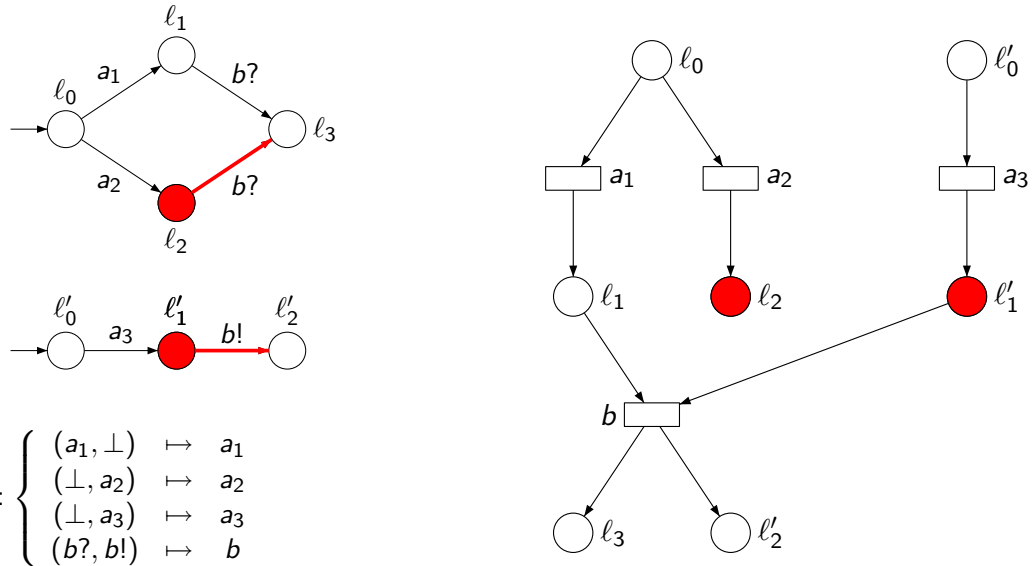

$f:\left\{\begin{array}{rlr}\left(a_{1}, \perp\right) & \mapsto a_{1} \\ \left(\perp, a_{2}\right) & \mapsto a_{2} \\ \left(\perp, a_{3}\right) & \mapsto a_{3} \\ (b ?, b !) & \mapsto b\end{array}\right.$ 


\section{Example of the unfolding of a discrete system}
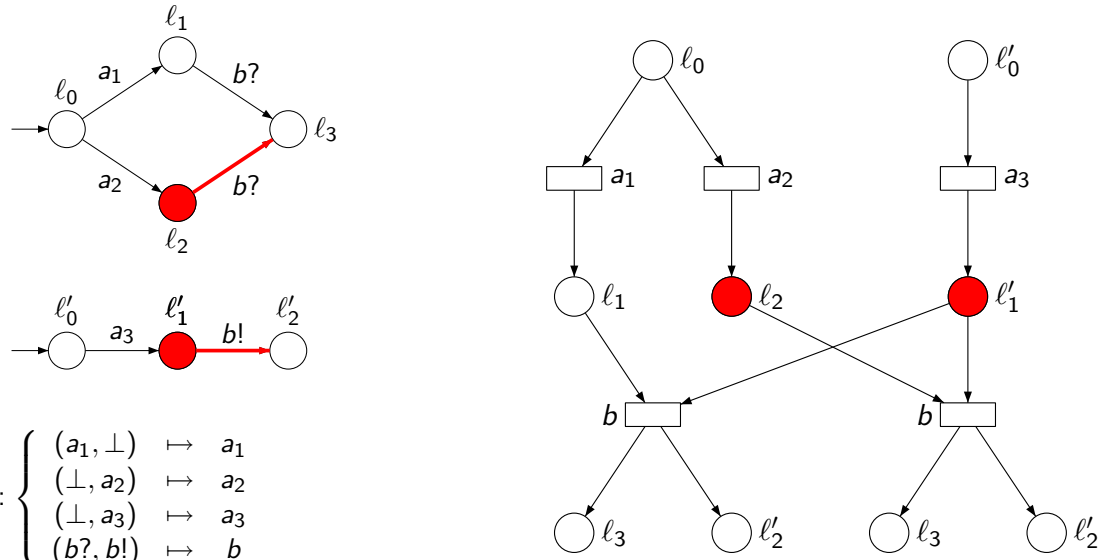

$f:\left\{\begin{array}{rlr}\left(a_{1}, \perp\right) & \mapsto a_{1} \\ \left(\perp, a_{2}\right) & \mapsto a_{2} \\ \left(\perp, a_{3}\right) & \mapsto a_{3} \\ (b ?, b !) & \mapsto & b\end{array}\right.$ 


\section{Example of the unfolding of a discrete system}
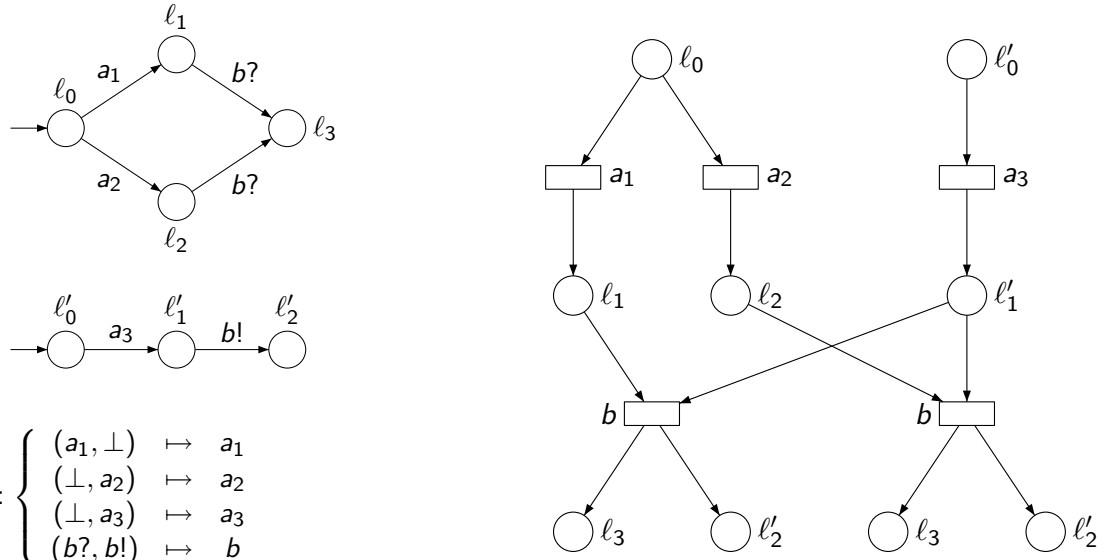

$f:\left\{\begin{array}{rlr}\left(a_{1}, \perp\right) & \mapsto a_{1} \\ \left(\perp, a_{2}\right) & \mapsto a_{2} \\ \left(\perp, a_{3}\right) & \mapsto a_{3} \\ (b ?, b !) & \mapsto & b\end{array}\right.$ 


\section{Example of the unfolding of a discrete system}

Some definitions:

Causal relation $<$ if $p \in{ }^{\bullet} t$, then $p<t$ if $p \in t^{\bullet}$, then $t<p$

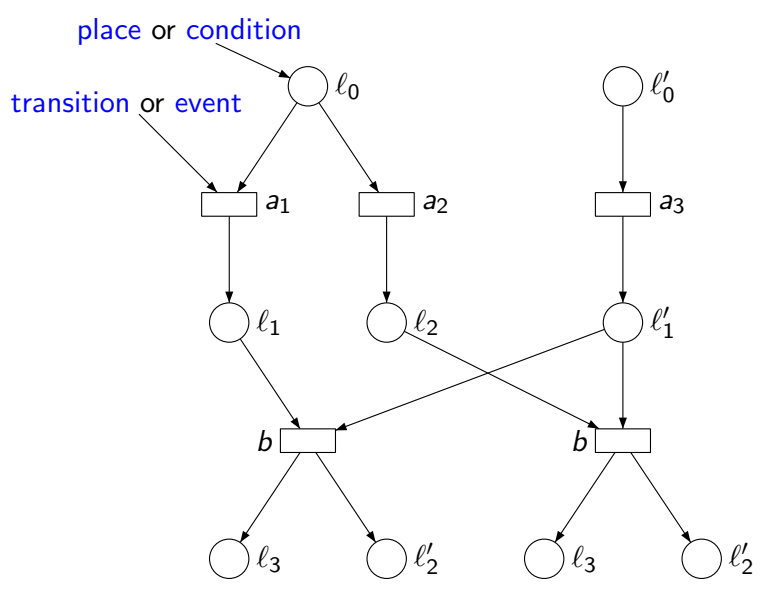




\section{Example of the unfolding of a discrete system}

Some definitions:

Causal relation $<$ if $p \in{ }^{\bullet} t$, then $p<t$ if $p \in t^{\bullet}$, then $t<p$

Non-branching process subnet corresponding to an execution

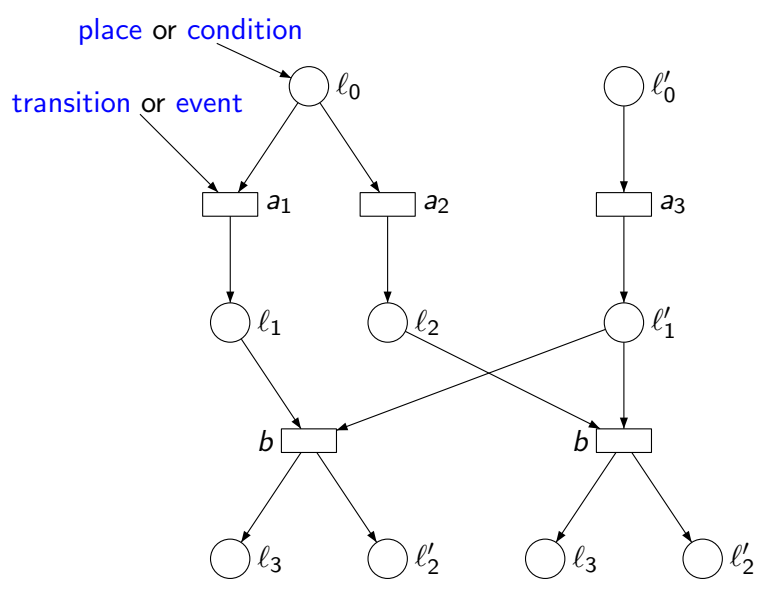




\section{Example of the unfolding of a discrete system}

Some definitions:

Causal relation $<$ if $p \in{ }^{\bullet} t$, then $p<t$ if $p \in t^{\bullet}$, then $t<p$

Non-branching process subnet corresponding to an execution

Nbp associated with an event minimal causal past

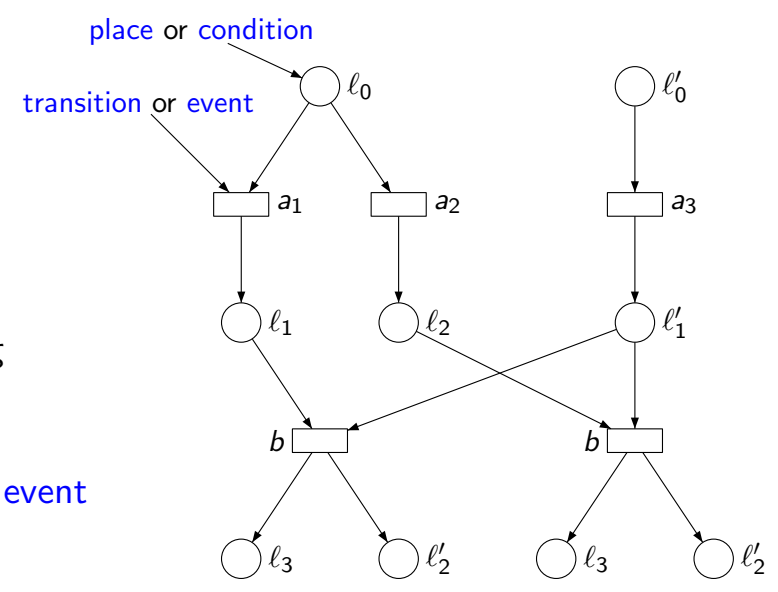




\section{Example of the unfolding of a discrete system}

Some definitions:

Causal relation $<$ if $p \in{ }^{\bullet} t$, then $p<t$ if $p \in t^{\bullet}$, then $t<p$

Non-branching process subnet corresponding to an execution

Nbp associated with an event minimal causal past

Cut of a nbp $C$ $\left(\operatorname{Min} \cup C^{\bullet}\right) \backslash \bullet C$

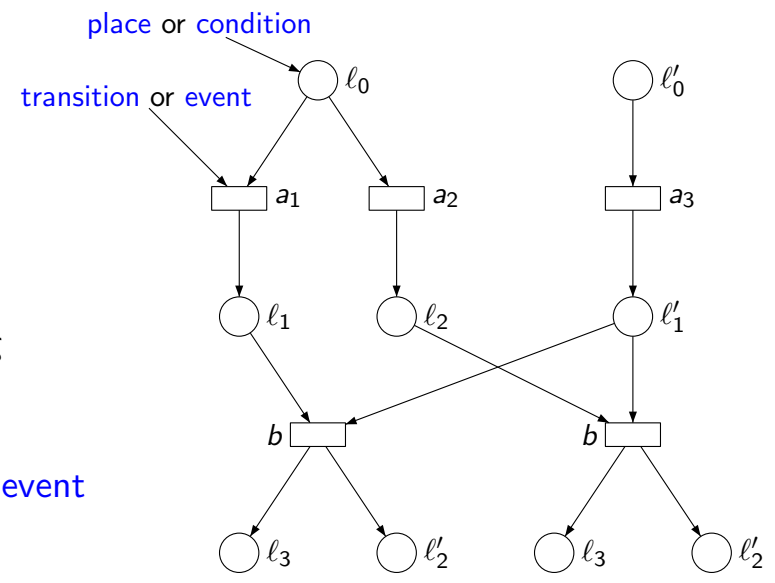


(1) Generalities

\section{- Networks of timed automata \\ - Unfolding of discrete-event systems}

(2) Discrete Structure of our Unfolding

- Clocks as shared variables

- Invariants

- An example

Adding Time

- Timed non-branching process

- A first timed unfolding

- Computation via local zones

Finite and complete prefix 


\section{Clocks as shared variables}

- Clocks induce dependencies betw. a priori indep. transitions

- Examples:

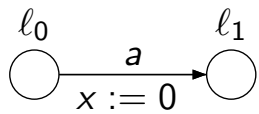

$a$ and $b$ are dependent

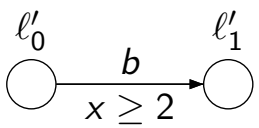

- Consider clocks as shared variables:

- Test as reading

- Reset as writing

Remark: related to [ICALP'06], [Srba], [Lugiez et al] 


\section{Clocks as shared variables}

- Clocks induce dependencies betw. a priori indep. transitions

- Examples:

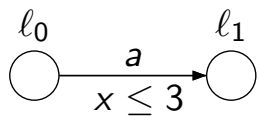

$a$ and $b$ are independent

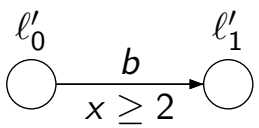

- Consider clocks as shared variables:

- Test as reading

- Reset as writing

Remark: related to [ICALP'06], [Srba], [Lugiez et al] 


\section{Test as reading}

Read Arcs: $t_{1} \square \longrightarrow \underbrace{}_{p} \longrightarrow t_{3} \quad \begin{aligned} & p \in t_{1} \bullet \wedge p \in{ }^{\circ} t_{2} \Rightarrow t_{1}<t_{2} \\ & p \in{ }^{\circ} t_{2} \wedge p \in{ }^{\circ} t_{3} \Rightarrow t_{2}<t_{3}\end{aligned}$

$\rightarrow$ Leads to another notion of unfolding: [Vogler et al, Winkovski]

- More difficult to define

- Increase significantly the concurrency relation
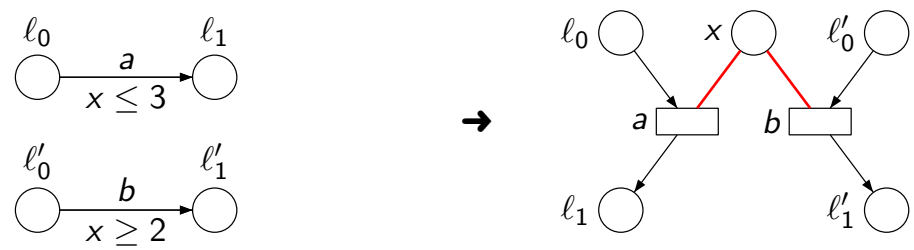

$a$ and $b$ are concurrent 


\section{Reset as writing}
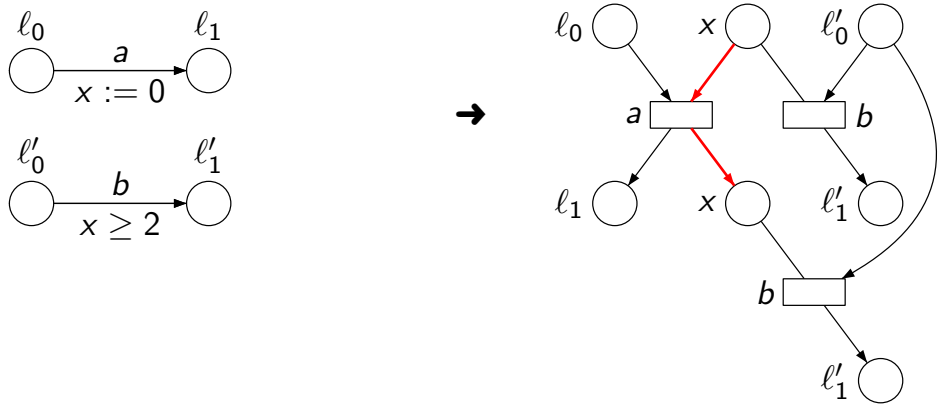

- $a$ and $b$ are dependent,

- there are two occurrences of the transition $b$. 


\section{Invariants}

- An invariant of any process may affect the behavior of other processes:

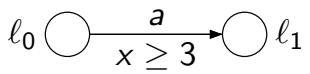

$\ell_{0}^{\prime} y \leq 2$
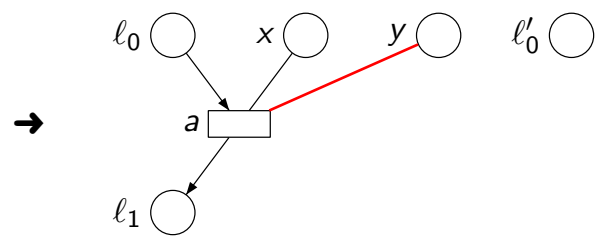

- For every clock $x$ appearing in an invariant, and for every event $t$, we require:

$$
x \in{ }^{\circ} t \cup^{\bullet} t
$$

- This may have bad consequences on the causal relation. 


\section{Invariants (2)}

-What happens if a transition modifies an invariant?

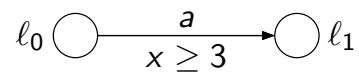

$\ell_{0}^{\prime} x \leq 2 \stackrel{b}{\longrightarrow x \leq 4} \ell_{1}^{\prime}$

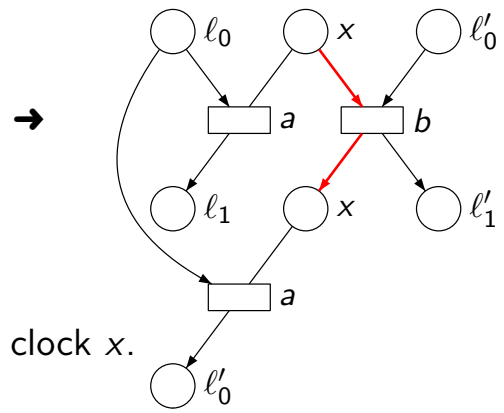

- To a condition encoding a clock $x$ corresponds informally a set of constraints satisfied by $x$. 


\section{Example}

$\bigcirc y \quad \bigcirc \ell_{0} \bigcirc \ell_{0}^{\prime} \bigcirc x \bigcirc \ell_{0}^{\prime \prime}$
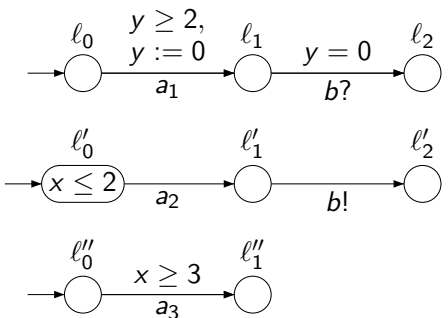

$f:\left\{\begin{array}{rlr}\left(a_{1}, \perp, \perp\right) & \mapsto a_{1} \\ \left(\perp, a_{2}, \perp\right) & \mapsto a_{2} \\ \left(\perp, \perp, a_{3}\right) & \mapsto a_{3} \\ (b ?, b !, \perp) & \mapsto b\end{array}\right.$ 


\section{Example}
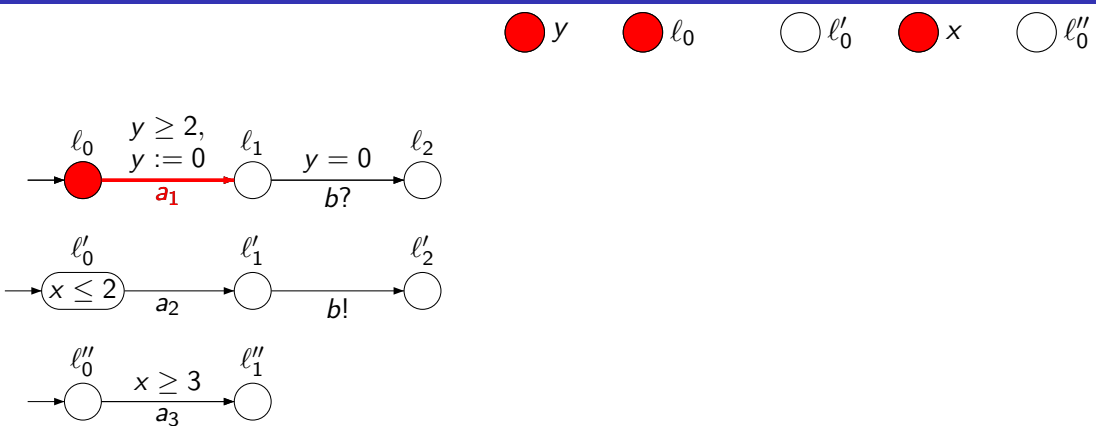

$f:\left\{\begin{array}{lll}\left(a_{1}, \perp, \perp\right) & \mapsto & a_{1} \\ \left(\perp, a_{2}, \perp\right) & \mapsto & a_{2} \\ \left(\perp, \perp, a_{3}\right) & \mapsto & a_{3} \\ (b ?, b !, \perp) & \mapsto & b\end{array}\right.$ 


\section{Example}
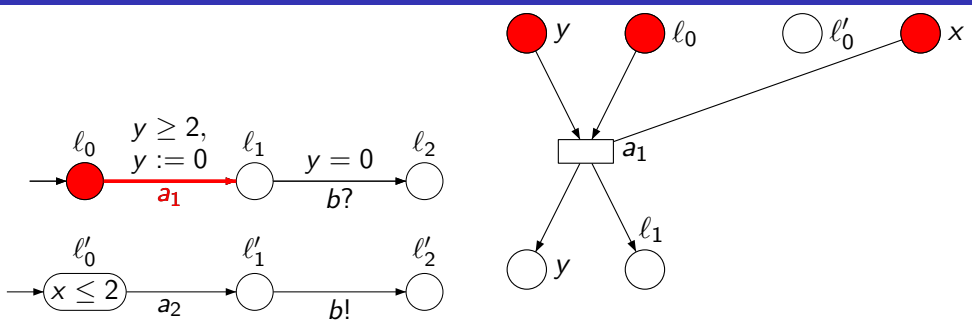

$\bigcirc \ell_{0}^{\prime \prime}$

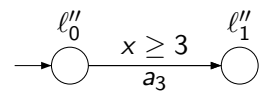

$f:\left\{\begin{array}{lll}\left(a_{1}, \perp, \perp\right) & \mapsto & a_{1} \\ \left(\perp, a_{2}, \perp\right) & \mapsto & a_{2} \\ \left(\perp, \perp, a_{3}\right) & \mapsto & a_{3} \\ (b ?, b !, \perp) & \mapsto & b\end{array}\right.$ 


\section{Example}
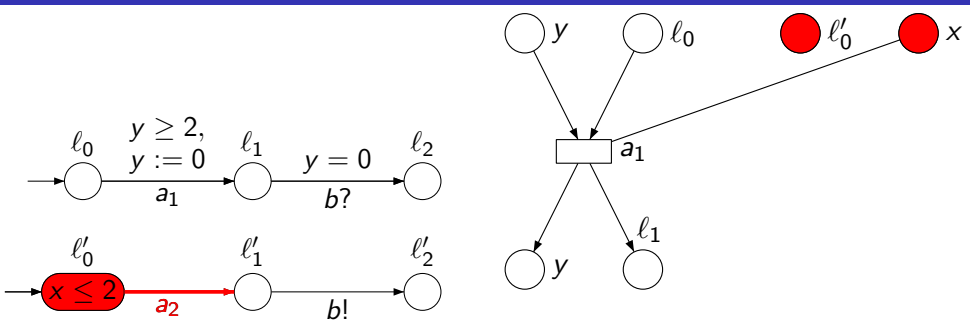

$\bigcirc \ell_{0}^{\prime \prime}$

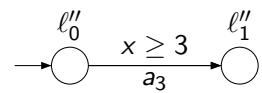

$f:\left\{\begin{array}{lll}\left(a_{1}, \perp, \perp\right) & \mapsto & a_{1} \\ \left(\perp, a_{2}, \perp\right) & \mapsto & a_{2} \\ \left(\perp, \perp, a_{3}\right) & \mapsto & a_{3} \\ (b ?, b !, \perp) & \mapsto & b\end{array}\right.$ 


\section{Example}
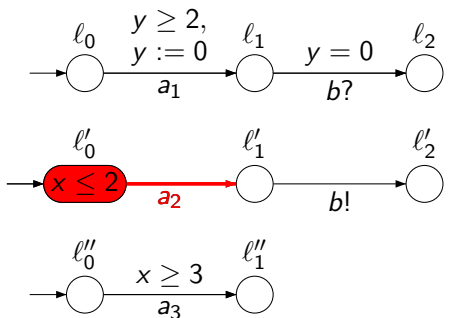

$f:\left\{\begin{array}{lll}\left(a_{1}, \perp, \perp\right) & \mapsto & a_{1} \\ \left(\perp, a_{2}, \perp\right) & \mapsto & a_{2} \\ \left(\perp, \perp, a_{3}\right) & \mapsto & a_{3} \\ (b ?, b !, \perp) & \mapsto & b\end{array}\right.$

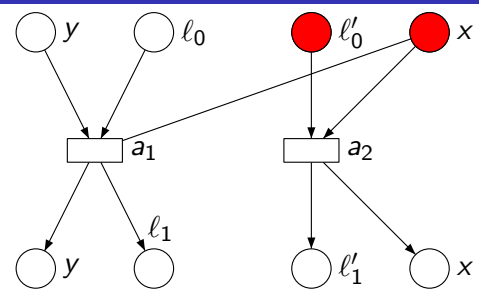

$\bigcirc \ell_{0}^{\prime \prime}$ 


\section{Example}
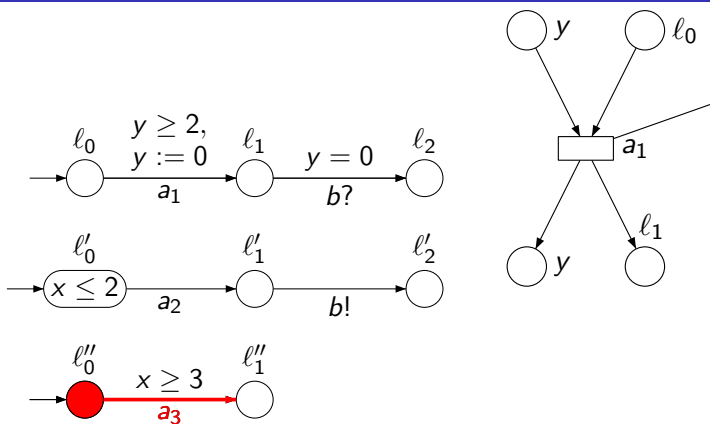

$f:\left\{\begin{array}{lll}\left(a_{1}, \perp, \perp\right) & \mapsto & a_{1} \\ \left(\perp, a_{2}, \perp\right) & \mapsto & a_{2} \\ \left(\perp, \perp, a_{3}\right) & \mapsto & a_{3} \\ (b ?, b !, \perp) & \mapsto & b\end{array}\right.$ 


\section{Example}
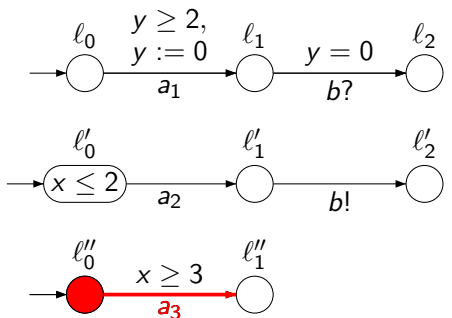

$f:\left\{\begin{array}{lll}\left(a_{1}, \perp, \perp\right) & \mapsto & a_{1} \\ \left(\perp, a_{2}, \perp\right) & \mapsto & a_{2} \\ \left(\perp, \perp, a_{3}\right) & \mapsto & a_{3} \\ (b ?, b !, \perp) & \mapsto & b\end{array}\right.$

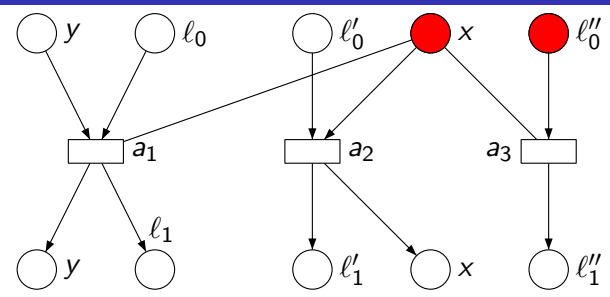




\section{Example}
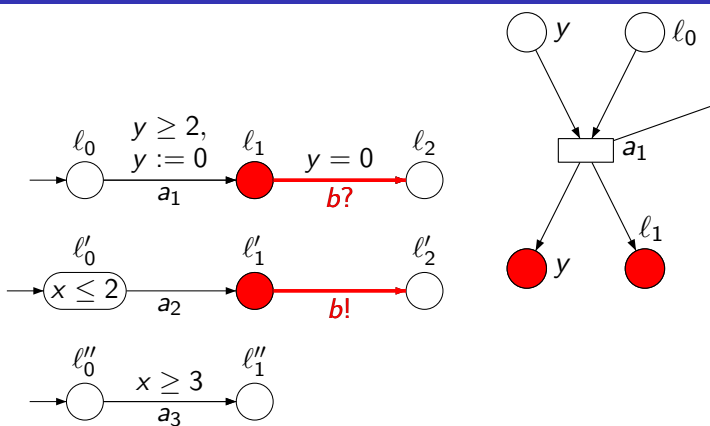

$f:\left\{\begin{array}{lll}\left(a_{1}, \perp, \perp\right) & \mapsto & a_{1} \\ \left(\perp, a_{2}, \perp\right) & \mapsto & a_{2} \\ \left(\perp, \perp, a_{3}\right) & \mapsto & a_{3} \\ (b ?, b !, \perp) & \mapsto & b\end{array}\right.$ 


\section{Example}
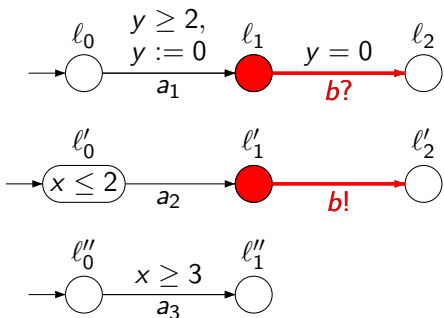

$f:\left\{\begin{array}{rlr}\left(a_{1}, \perp, \perp\right) & \mapsto & a_{1} \\ \left(\perp, a_{2}, \perp\right) & \mapsto & a_{2} \\ \left(\perp, \perp, a_{3}\right) & \mapsto & a_{3} \\ (b ?, b !, \perp) & \mapsto & b\end{array}\right.$

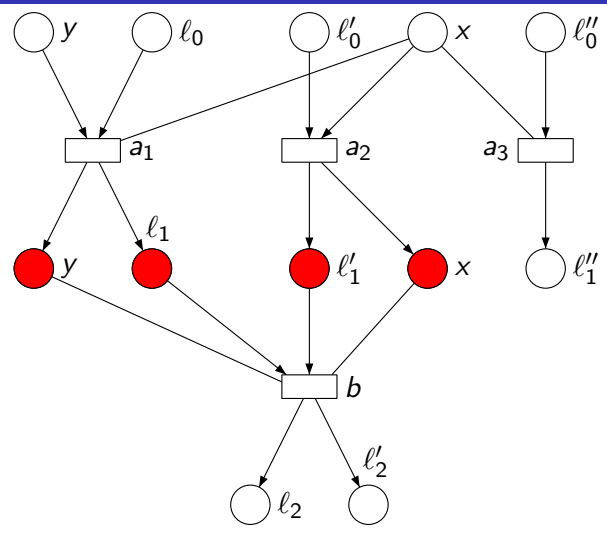




\section{Example}
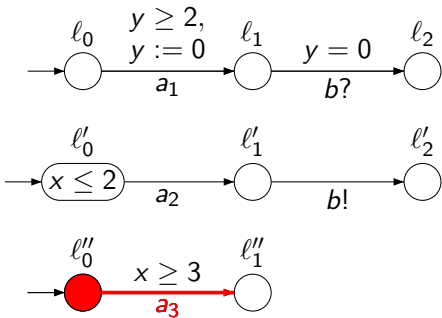

$f:\left\{\begin{array}{lll}\left(a_{1}, \perp, \perp\right) & \mapsto & a_{1} \\ \left(\perp, a_{2}, \perp\right) & \mapsto & a_{2} \\ \left(\perp, \perp, a_{3}\right) & \mapsto & a_{3} \\ (b ?, b !, \perp) & \mapsto & b\end{array}\right.$

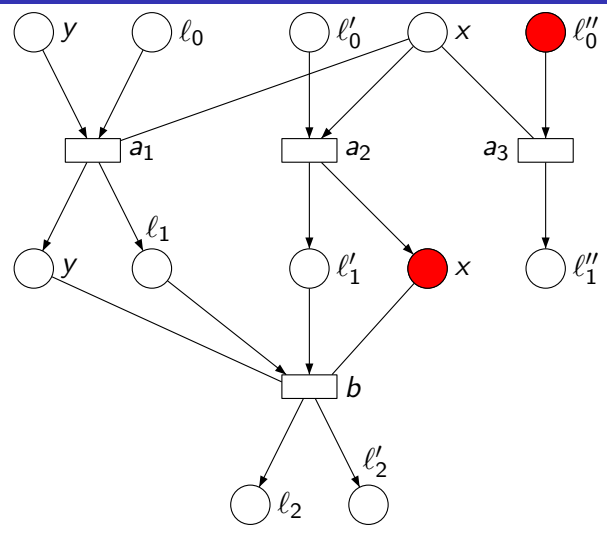




\section{Example}
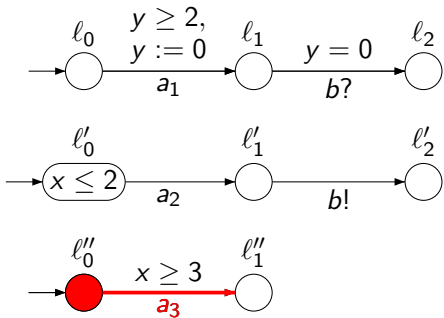

$f:\left\{\begin{array}{rlr}\left(a_{1}, \perp, \perp\right) & \mapsto & a_{1} \\ \left(\perp, a_{2}, \perp\right) & \mapsto & a_{2} \\ \left(\perp, \perp, a_{3}\right) & \mapsto & a_{3} \\ (b ?, b !, \perp) & \mapsto & b\end{array}\right.$

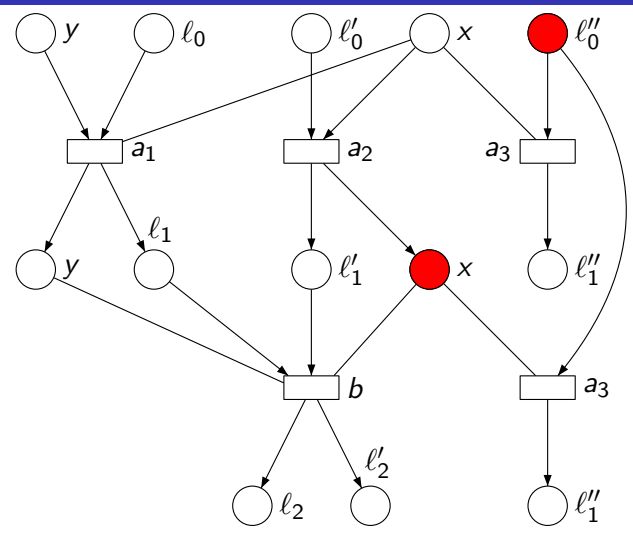




\section{Example}
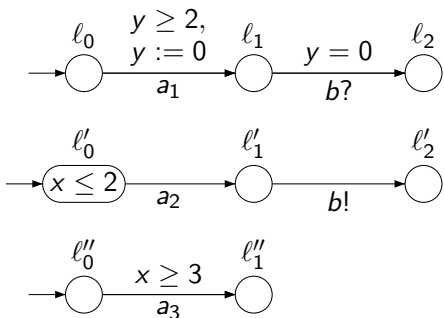

$f:\left\{\begin{array}{lll}\left(a_{1}, \perp, \perp\right) & \mapsto & a_{1} \\ \left(\perp, a_{2}, \perp\right) & \mapsto & a_{2} \\ \left(\perp, \perp, a_{3}\right) & \mapsto & a_{3} \\ (b ?, b !, \perp) & \mapsto & b\end{array}\right.$

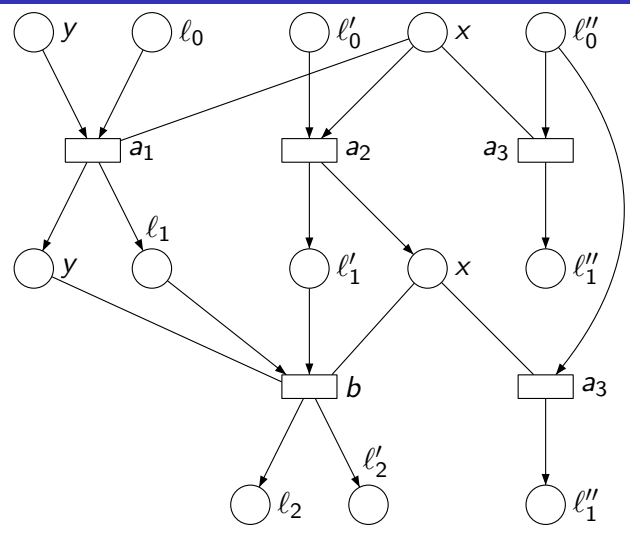




\section{Example}
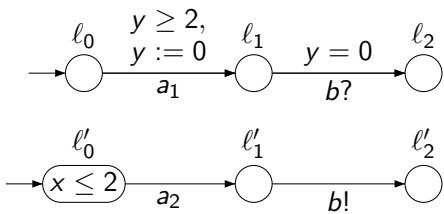

$\rightarrow \bigcirc_{a_{3}}^{\ell_{0}^{\prime \prime}} x \geq 3$

$f:\left\{\begin{array}{rlr}\left(a_{1}, \perp, \perp\right) & \mapsto & a_{1} \\ \left(\perp, a_{2}, \perp\right) & \mapsto & a_{2} \\ \left(\perp, \perp, a_{3}\right) & \mapsto & a_{3} \\ (b ?, b !, \perp) & \mapsto & b\end{array}\right.$

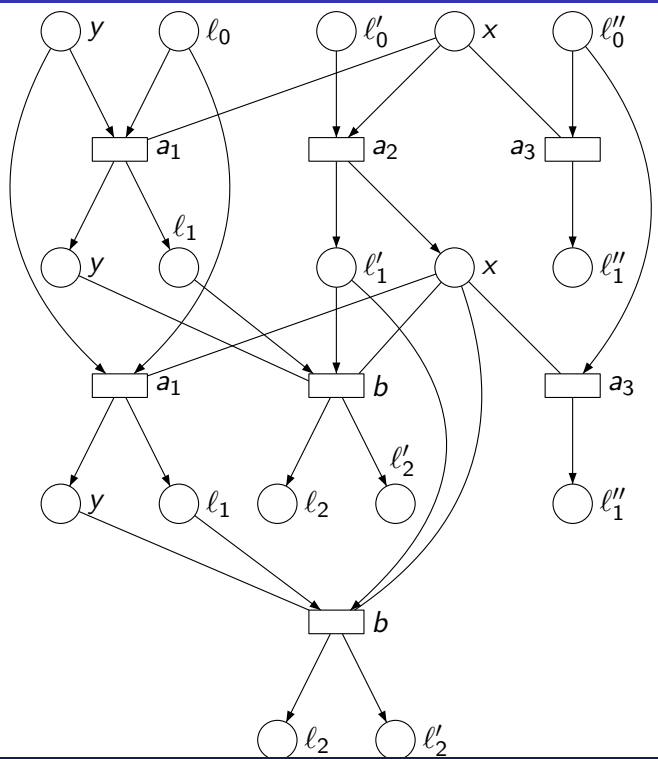

Pierre-Alain Reynier 


\section{Generalities}

- Networks of timed automata

- Unfolding of discrete-event systems

(2) Discrete Structure of our Unfolding

- Clocks as shared variables

- Invariants

- An example

(3) Adding Time

- Timed non-branching process

- A first timed unfolding

- Computation via local zones

Finite and complete prefix 


\section{Timed Non-branching Process}

We attach timing informations to nodes:

- For transitions:

$>\mathbf{d}$ : date of firing

- For locations:

- $\mathbf{d}_{\mathbf{b}}$ : date of birth (= production)

- $\mathbf{d}_{\mathrm{e}}$ : date of end (= consumption)

- For clocks:

- $\mathbf{d}_{\mathbf{b}}$ : date of birth (= production)

$-\mathbf{d}_{\mathbf{e}}$ : date of end (= consumption)

- $\mathbf{d}_{\mathbf{r}}$ : date of last reset (to compute clock's value)

$\rightarrow$ These are absolute dates 


\section{From timed sequences to timed nbp}

A timed sequence $\nu=\nu^{\prime} \cdot(d, a)$
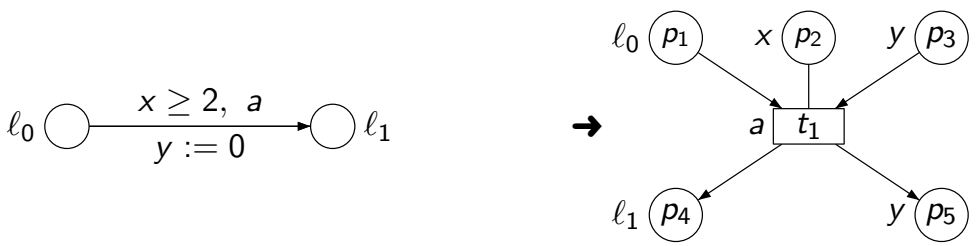

We define dates as follows:

$\mathbf{d}\left(t_{1}\right)=d$

$\mathbf{d}_{\mathbf{e}}\left(p_{1}\right)=\mathbf{d}_{\mathbf{e}}\left(p_{3}\right)=d$

$\mathbf{d}_{\mathbf{b}}\left(p_{4}\right)=\mathbf{d}_{\mathbf{b}}\left(p_{5}\right)=\mathbf{d}_{\mathbf{r}}\left(p_{5}\right)=d$ 


\section{Feasible Timed Non-branching Process}

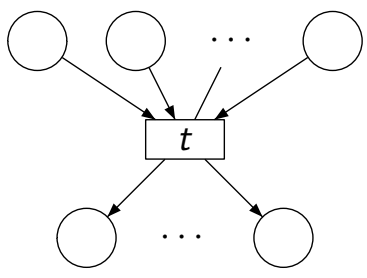

Causal (in)equations:

- $\forall p \in t^{\bullet}, \mathbf{d}_{\mathbf{b}}(p)=\mathbf{d}(t)$

- $\forall p \in{ }^{\bullet} t, \mathbf{d}_{\mathbf{e}}(p)=\mathbf{d}(t)$

- $\forall p \in{ }^{\circ} t, \mathbf{d}_{\mathbf{b}}(p) \leq \mathbf{d}(t) \leq \mathbf{d}_{\mathbf{e}}(p)$

- $\forall p \in \operatorname{Min}, \mathbf{d}_{\mathbf{b}}(p)=\mathbf{d}_{\mathbf{r}}(p)=0$ for any clock $x$, let us denote:

$$
\begin{aligned}
& v(x)=\mathbf{d}(t)-\mathbf{d}_{\mathbf{r}}\left(p_{x}^{-}\right) \\
& v^{\prime}(x)=\mathbf{d}(t)-\mathbf{d}_{\mathbf{r}}\left(p_{x}^{+}\right)
\end{aligned}
$$

Timed (in)equations:

- $g(t)\left[\{x \leftarrow v(x)\}_{x \in X]}\right.$

- $\bigwedge_{\ell \in L(t)} \ln v(\ell)\left[\{x \leftarrow v(x)\}_{x \in X}\right]$

- $\bigwedge_{x \in R(t)} v^{\prime}(x)=0$

- $\bigwedge_{x \in \text { Redefined }(t)} v^{\prime}(x)=v(x)$ 


\section{A first timed Unfolding}

- A timed non-branching process is feasible iff it satisfies previous equations.

Proposition

(i) The timed nbp associated with a timed sequence is feasible,

(ii) If a timed $n b p$ is feasible, then it admits a timed sequence.

- If we consider the previous equations as zones (symbolic representation for valuations), then we get:

\section{Theorem}

The resulting timed unfolding contains exactly all the timed sequences of the NTA : it defines a symbolic unfolding for NTA. 


\section{Computation via local zones}

- Drawback of the previous unfolding: the size of zones increases while unfolding.

- Idea: keep only the part of the zone related to the cut $\rightarrow$ zones of bounded size $(2 n+3|X|)$.

- Interest: sufficient to extend a nbp.

- Drawback: not sufficient to "merge" two nbps.

$\triangleright$ Details 


\section{Computation via local zones (2)}

- Using a topological sort, we compute local zones "slice by slice" (slice = set of concurrent events).

- We obtain a way to compute a timed unfolding of an NTA, in which zones attached to events are of bounded size.

\section{Theorem}

We can compute the timed unfolding $\mathcal{T}(\mathcal{A})$ with local zones $\left(Z_{t}\right)_{t}$ verifying:

- $t$ appears in $\mathcal{T}(\mathcal{A})$ iff there exists a timed sequence whose nbp is the one of $t$,

- $Z_{t}$ characterizes the set of values reachable by timed sequences along the $n b p$ of $t$ 
(1) Generalities

- Networks of timed automata

- Unfolding of discrete-event systems

(2) Discrete Structure of our Unfolding

- Clocks as shared variables

- Invariants

- An example

Adding Time

- Timed non-branching process

- A first timed unfolding

- Computation via local zones

(4) Finite and complete prefix 


\section{Objective}

- Limit the unfolding to a finite prefix which contains full information about the reachable states.

- In the untimed framework:

$$
\text { e cut-off } \Longleftrightarrow \exists e^{\prime} \prec e \text { s.t. }\left\{\begin{array}{l}
\lambda(e)=\lambda\left(e^{\prime}\right) \\
\lambda(\operatorname{Cut}(e))=\lambda\left(\operatorname{Cut}\left(e^{\prime}\right)\right)
\end{array}\right.
$$

Then prune the subtree rooted in $e$.

- In the timed framework, we have to compare zones! (inclusion) 


\section{How to compare zones}

- Compute relativization of zone $Z_{t}$ w.r.t. date $\mathbf{d}(t)$ of $t$ : (operation : $\forall \mathbf{d}, \mathbf{d}:=\mathbf{d}(t)-\mathbf{d}$ )

- But these zones may be unbounded $\rightarrow$ does not ensure termination!

- Favorable case: "bounded" TA $=$ TA such that :

- clock values are bounded,

- time elapsed in any location is bounded.

$\rightarrow$ finitely many zones $\Rightarrow$ Termination!

- General case: Enforce global synchronization to reinitialize the unfolding, and use extrapolation on "clock zones". 


\section{Synchronized events}

Define the clock zones Test $_{t}$ obtained from $Z_{t}$ by performing:

- Relativization w.r.t. variable $\mathbf{d}(t)$

- Projection on variables $\mathbf{d}_{\mathbf{r}}($.

- Extrapolation

Synchronized events: (chosen among unavoidable edges) Perform global synchronization, and reinitialize the unfolding (consume all places of the cut, and produce them back)

Lemma (Forgettable Past)

Let $t \in S E$.

It is equivalent to extend the $n b p$ of $t$ and to build a nbp from Cut $([t])$ which initially satisfies Test $_{t}$.

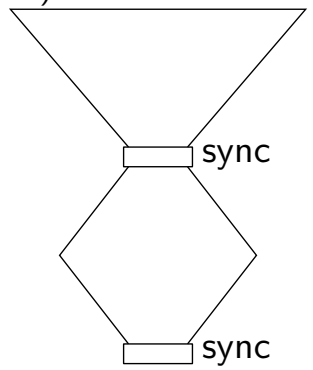




\section{Algorithm for finite prefix}

- We consider an adequate order $\prec$ on SE (see [Esparza et al])

- Cut-off event :

$$
e \in S E \text { cut-off } \Longleftrightarrow \exists e^{\prime} \prec e \text { s.t. }\left\{\begin{array}{l}
\lambda(e)=\lambda\left(e^{\prime}\right) \\
\lambda(\operatorname{Cut}(e))=\lambda\left(\operatorname{Cut}\left(e^{\prime}\right)\right) \\
\text { Test }_{e} \subseteq \text { Test }_{e^{\prime}}
\end{array}\right.
$$

Theorem

This algorithm terminates and produces a finite and complete prefix. 
(1) Generalities

- Networks of timed automata

- Unfolding of discrete-event systems

(2) Discrete Structure of our Unfolding

- Clocks as shared variables

- Invariants

- An example

Adding Time

- Timed non-branching process

- A first timed unfolding

- Computation via local zones

(4) Finite and complete prefix

(5) Conclusion 


\section{Conclusion}

- Definition of a timed unfolding for NTA

- Computation using "local zones"

- Construction of a finite and complete prefix

Further work:

- Reduce dependencies due to invariants

- Reduce the number of variables $\left(\mathbf{d}_{\mathbf{b}}, \mathbf{d}_{\mathbf{e}}, \mathbf{d}_{\mathbf{r}}\right)$

- Try to avoid synchronized edges

- Extend the set of properties we can verify

- Implementation 


\section{A last remark}

There were two papers at ATVA'06:

- "Timed Unfoldings For Networks of Timed Automata" (BHR)

- "Symbolic Unfoldings For Networks of Timed Automata" (Cassez, Chatain, Jard)

The two contributions are close but there are important differences:

- they propose a way to define the unfolding, but do not consider its "efficient" construction,

- this allows them to define a "smaller" object,

- they make some stronger assumptions on the system (no deadlocks, no shared clocks).

$\rightarrow$ We are currently trying to bring closer the two approaches. 
Thanks for your attention! 


\section{Extend the unfolding}

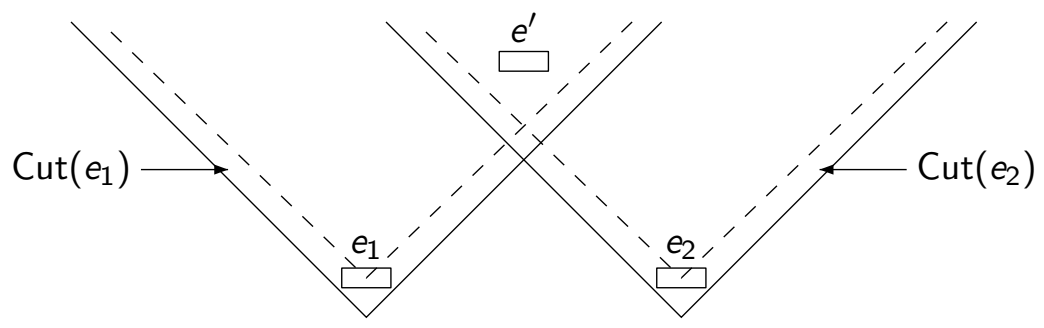

Firing $e_{1}$ implies $\mathbf{d}\left(e^{\prime}\right)<1$

Firing $e_{2}$ implies $\mathbf{d}\left(e^{\prime}\right)>1$

But $e^{\prime}$ does not appear in $\operatorname{Cut}\left(e_{1}\right) \cup \operatorname{Cut}\left(e_{2}\right) \quad$ !

$\rightarrow$ Taking $Z_{e_{1}} \wedge Z_{e_{2}}$ is not enough precise... 


\section{Computing by slices}

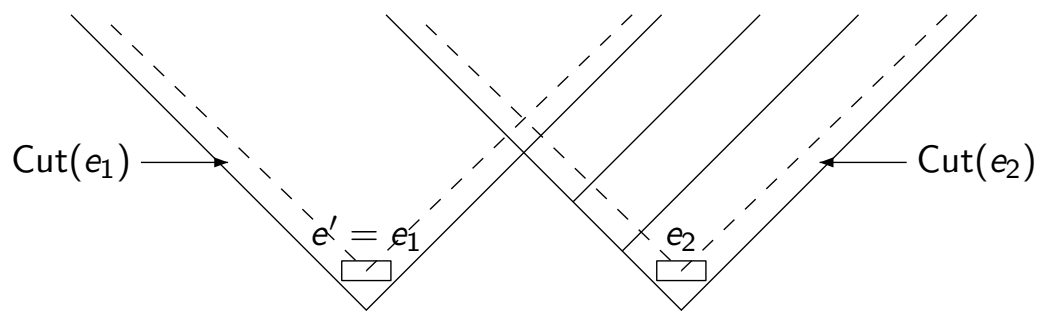

(1) Compute the starting event $e^{\prime}$

(2) Sort by slices (sets of concurrent events) the elements of $\left(\left[e_{1}\right] \cup\left[e_{2}\right]\right) \backslash\left[e^{\prime}\right]$

(3) Extend $Z_{e^{\prime}}$, slice by slice.

$\triangleright$ Back 